Nobuhide Ueki • Naohiko Seki • Kazuhiro Yano

Toshiyuki Saito · Yasuhiko Masuho • Masa-aki Muramatsu

\title{
Isolation and chromosomal assignment of a human gene encoding protein inhibitor of activated STAT3 (PIAS3)
}

\begin{abstract}
Mouse PIAS3 (protein inhibitor of activated STAT3) is a specific inhibitor of STAT3 that downregulates its signaling pathway. Here we report the isolation and chromosome mapping of the human PIAS3 gene. Human PIAS3 cDNA encoded a predicted protein of 619 aa which has $83 \%$ overall amino acid identity to the mouse counterpart. Based on polymerase chain reaction assisted analysis of a human/ rodent mono-chromosomal hybrid cell panel and a radiation hybrid mapping panel, the human PIAS3 gene was mapped to the chromosome 1q21 region. Mapping of a crucial gene in modulating the STAT3 signaling pathway may provide new clues to the understanding of malignancies or genetic disorders caused by this chromosome region.
\end{abstract}

Key words STAT3 - PIAS3 - Cytokine - Transcription factor $\cdot$ Hematopoiesis $\cdot 1 \mathrm{q} 21$

\section{Introduction}

STAT (signal transducer and activator of transcription) proteins are latent cytoplasmic transcription factors that become activated by tyrosine phosphorylation in response to cytokine stimulation (Ihle and Kerr 1995). Tyrosine phosphorylated STATs undergo dimerization and translo-

N. Ueki $\cdot$ K. Yano $\cdot$ Y. Masuho $\cdot$ M. Muramatsu $(\square)$

Helix Research Institute, 1532-3 Yana, Kisarazu, Chiba 292-0812, Japan

Tel. +81-438-3951; Fax +81-438-3952

e-mail:mmasaaki@hri.co.jp

N. Seki

Kazusa DNA Research Institute, Chiba, Japan

N. Seki · T. Saito

Genome Research Group, National Institute Radiological Sciences, Chiba, Japan

M. Muramatsu

Department of Biological Cybernetics, Medical Research Institute,

Tokyo Medical Dental University, Tokyo, Japan cate into the nucleus to activate specific set of genes. Seven members of the STAT protein family have been isolated, which have distinct functions in cytokine signaling (Leonard and O'Shea 1998). STAT3 is activated by cytokines such as interlukin-6 (IL-6), leukemia inhibitory factor (LIF), oncostatin $\mathrm{M}$, granulocyte colony-stimulating factor (G-CSF), epidermal growth factor (EGF), and leptin (Akira et al. 1994, Zhong et al. 1994, Vaisse et al. 1996). Recently, a family of proteins named PIAS (protein inhibitor of activated STAT), which can bind to STATs and inhibit their transcriptional activity, has been identified. Mouse PIAS1 and PIAS3 can specifically inhibit STAT1 and STAT3 signaling, respectively (Liu et al. 1998, Chung et al. 1997). In humans, PIAS1, as well as additional members PIASx $\alpha$, PIASx $\beta$, and PIASy, have also been identified (Liu et al 1998). However, the function of these new members still remains elusive. Here we report the isolation of human PIAS3 cDNA and its chromosomal location.

\section{Source and isolation of the human PIAS3 gene}

A partial cDNA clone (initially called HFB096) which had a sequence highly homologous to mouse PIAS3 was identified by a screening system for nuclear proteins (Ueki et al. 1998). Specific primers were designed according to the HFB096 sequence, and the 5' portion of the cDNA was obtained from a human fetal brain library, using GeneTrapper (GIBCO BRL, Gaithersburg, MD, USA). The nucleotide sequences of both strands were determined by a primer walking method and using an ABI377 sequencer (Perkin Elmer, Norwalk, CT, USA). The resultant cDNA was 2802 bp in length, with a poly A at the $3^{\prime}$ end, and contained an open reading frame (ORF) of 619 amino acids. The nucleotide sequence of the cDNA will appear in GenBank/EMBL/DDBJ databases under the accession number AB021868. The sequence analysis of the cDNA clone revealed that the predicted protein had an overall high homology to mouse PIAS3, and thus we renamed this clone human PIAS3. The alignments of the amino acid 


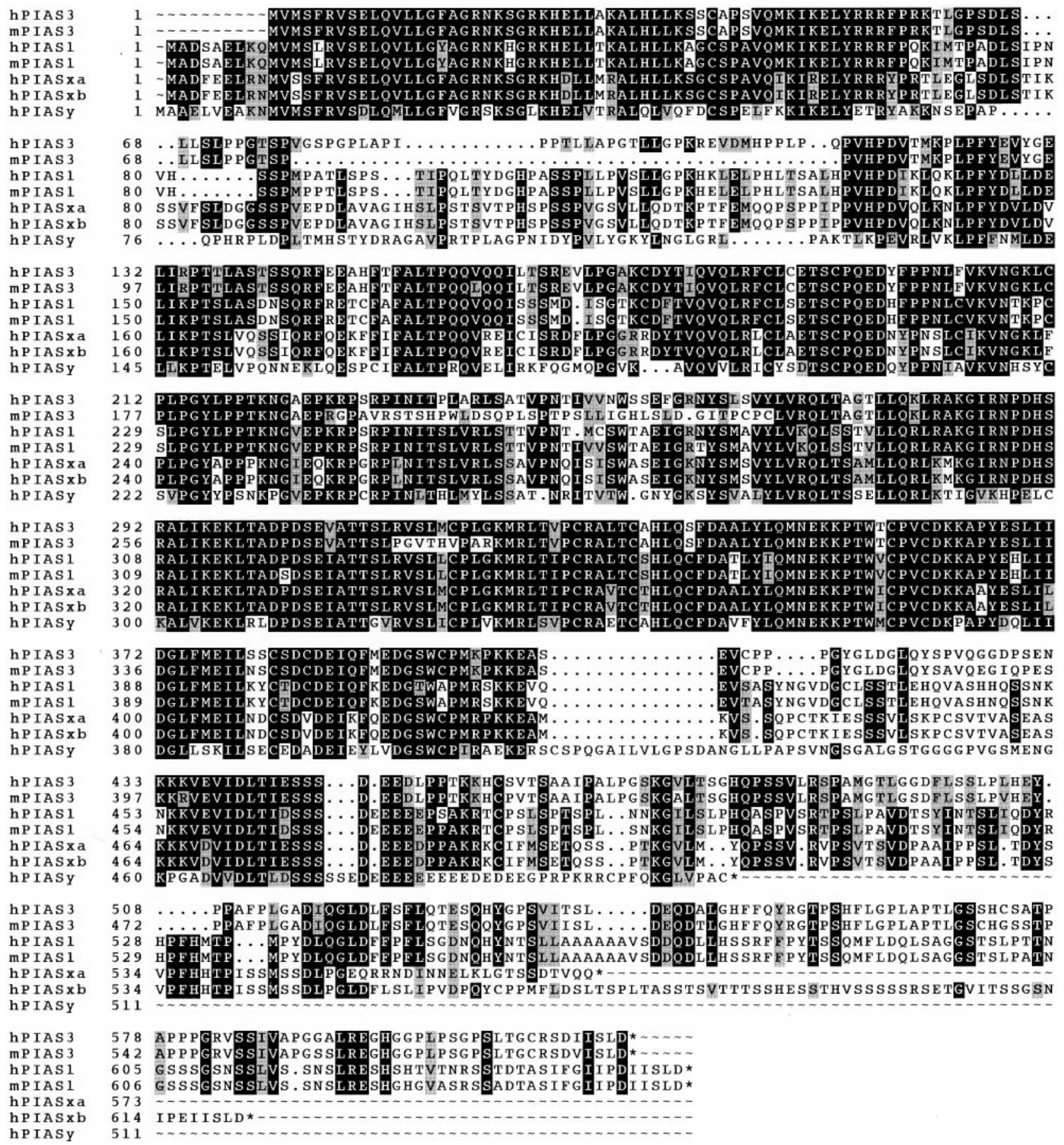

Fig. 1. Alignment of the PIAS family of proteins. The predicted amino acid sequences of human PIAS1 (hPIAS1; accession number, AF077951), mouse PIAS1 (mPIAS1; accession number, AF077950), human PIAS3 (hPIAS3; accession number, AB021868), mousePIAS3 (mPIAS3; accession number, AF034080), human PIASx $\alpha$ (hPIASxa; accession number, AF077953), human PIASxb (hPIASx $\beta$; accession number, AF077954) and human PIASy (hPIASy; accession number, AF077952)are shown. Identities are indicated by black background and similar residues are shadowed. Asterisks denote the termination codons sequences of hPIAS3, mPIAS3, hPIAS1, mPIAS1, hPIASx $\alpha$, hPIASx $\beta$, and hPIASy are shown in Fig. 1 . The amino acid identity of human and mouse PIAS3 proteins was $83 \%$, while hPIAS3 and hPIAS1, hPIASxa, hPIASxb, and hPIASy were $56 \%, 53 \%, 55 \%$, and $39 \%$ identical, respectively.

\section{Chromosomal mapping of the gene}

Chromosomal assignment of the human PIAS3 gene was done by polymerase chain reaction (PCR) analysis of a human/rodent somatic cell hybrid panel (National Institute 
Fig. 2. Chromosomal placement of the human PIAS3 gene at a relative distance to framework markers on the WICGR (Whitehead Institute for Biomedical Research/MIT Center for Genome Research) radiation hybrid map of the human genome (http://www.genome.wi. mit.edu/). The approximate corresponding cytogenetic location of the gene on the $1 \mathrm{q} 21$ region is indicated. Distances of the markers are in centirays (cR) and centimorgans (cM) from the top of the chromosome 1 linkage group

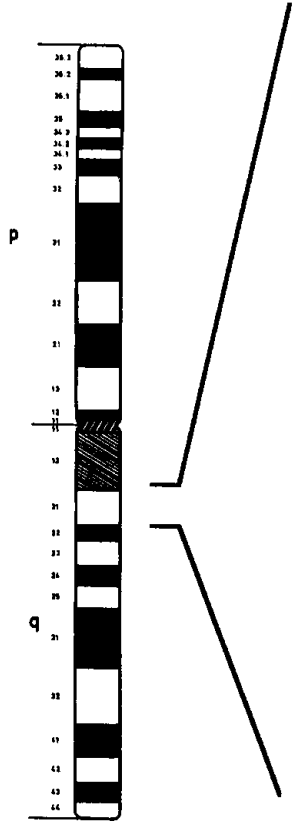

Chromosome 1

of General Medicine Service, Coriell Cell Repositories, Camden, NJ, USA) and a radiation hybrid panel (Genebridge 4, Research Genetics, Huntsville, AL, USA), as described previously (Seki et al, 1997). The hPIAS3specific PCR primers were designed at the $3^{\prime}$-untranslated region of the gene (5'-GAT TGG GAA GGA GGG CAC AGG-3', 5'-ACT TCC CCT GCC TCC TAC TCC-3') which would give rise to a PCR product with a size of 240 bp. The specific amplified product for humans was detected only from the hybrid containing human chromosome 1 (data not shown). Then, we performed further mapping analysis, using a radiation hybrid panel with the same primer set. Statistical analysis of the radiation hybrid data was performed using the RHMAPPER software package (http://www-genome.wi.mit.edu/cgi-bin/contig/ rhmapper.pl). The data vector for the hPIAS3 gene was $\begin{array}{llll}0001010001 & 0110000110 & 0000110110 & 0101000110\end{array}$ $\begin{array}{llll}1011101000 & 1001000000 & 0000101010 & 0101000001\end{array}$ 1100000101011 and the consequent report indicated that the gene was placed to $7.36 \mathrm{cR}$ distal from D1S442 $(\operatorname{lod}>3.0)$. The region including the marker was cytogenetically mapped to the 1q21 region (Lioumi et al. 1996) (Fig. 2).

\section{Discussion}

STAT3 is an essential transcription factor for the growth and development of hematopoietic cells (Minami et al. 1996). Thus, dysregulation of the STAT3 signaling cascade

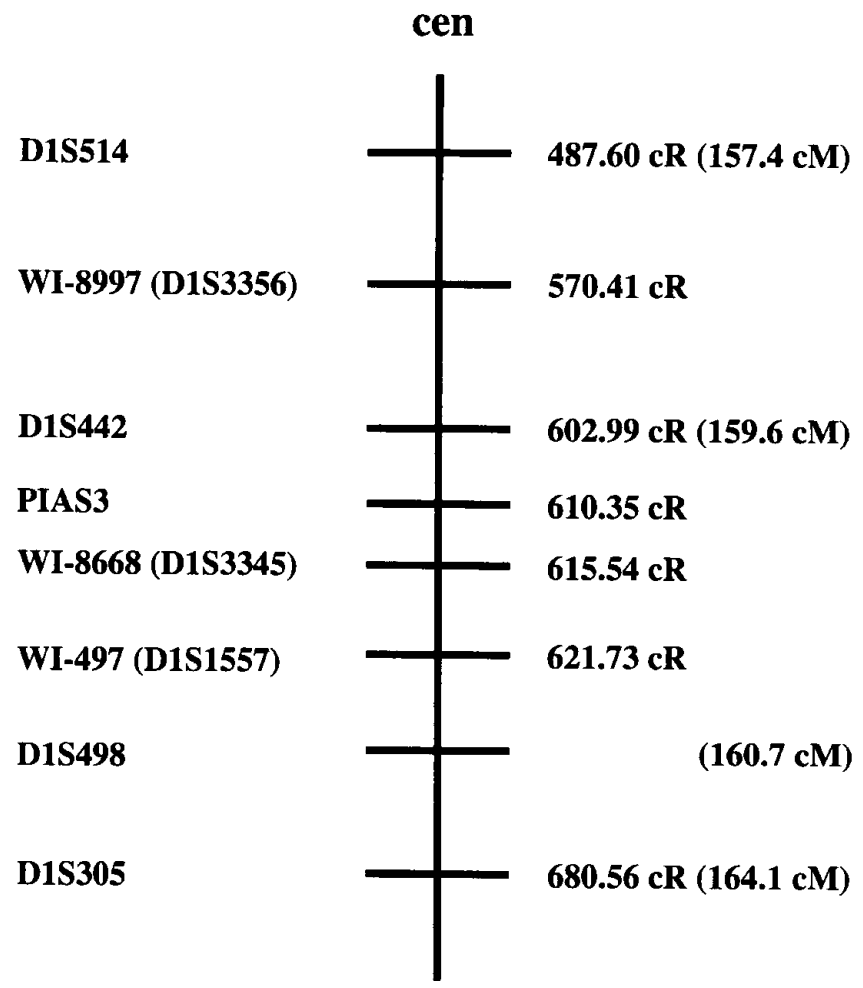

tel may lead to uncontrolled hematopoiesis. In fact, STAT3 is found to be constitutively activated in acute leukemia (Gouilleux-Gruart et al. 1997). However, no mutation in the STAT3 gene associated with human diseases has been found to date. Since PIAS3 is an inhibitor of STAT3, it is conceivable that dysregulation of PIAS3 may modulate STAT3 signaling. In this respect, it is noteworthy that the 1q21 region where hPIAS3 is mapped is associated with hematopoietic malignancies such as multiple myeloma (Keung et al. 1998) and large B-cell lymphoma (Rao et al. 1998). Our precise chromosomal positioning of the hPIAS3 gene may contribute toward the ongoing positional candidate approaches for the above-mentioned diseases linked to the chromosomal locus.

\section{References}

Akira S, Nishio Y, Inoue M, Wang XJ, Wei S, Matsusaka T, Yoshida K, Sudo T, Naruto M, Kishimoto T (1994) Molecular cloning of APRF, a novel IFN-stimulated gene factor 3 p91-related transcription factor involved in the gp130-mediated signaling pathway.Cell 77: 63-71

Chung CD, Liao J, Liu B, Rao X, Jay P, Berta P, Shuai K (1997) Specific inhibition of Stat3 signal transduction by PIAS3. Science 278: 1803-1805

Gouilleux-Gruart V, Debierre-Grockiego F, Gouilleux F, Capiod JC, Claisse JF, Delobel J, Prin L (1997) Activated Stat related transcription factors in acute leukemia. Leuk Lymphoma 28: 83-88

Ihle JN, Kerr IM (1995) Jaks and Stats in signaling by the cytokine receptor superfamily. Trends Genet 11: 69-74

Keung YK, Yung C, Wong JW, Shah F, Cobos E, Tonk V (1998) Unusual presentation of multiple myeloma with "jumping transloca- 
tion" involving 1q21. A case report and review of the literature. Cancer Genet Cytogenet 106: 135-139

Leonard WJ, O'Shea JJ (1998) Jaks and STATs: biological implications. Annu Rev Immunol 16: 293-322

Lioumi M, Olavesen MG, Nizetic D, Ragoussis J (1996) High-resolution YAC fragmentation map of 1q21. Genomics 49: 200-208

Liu B, Liao J, Rao X, Kushner SA, Chung CD, Chang DD, Shuai K (1998) Inhibition of Stat1-mediated gene activation by PIAS1. Proc Natl Acad Sci USA 95: 10 626-10 631

Minami M, Inoue M, Wei S, Takeda K, Matsumoto M, Kishimoto T, Akira S (1996) STAT3 activation is a critical step in gp130-mediated terminal differentiation and growth arrest of a myeloid cell line. Proc Natl Acad Sci USA 93: 3963-3966

Rao PH, Houldsworth J, Dyomina K, Parsa NZ, Cigudosa JC, Louie DC, Popplewell L, Offit K, Jhanwar SC, Chaganti RS (1998) Chro- mosomal and gene amplification in diffuse large B-cell lymphoma. Blood 92: 234-240

Seki N, Nimura Y, Ohira M, Saito T, Ichimiya S, Nomura N, Nakagawara A (1997) Identification and chromosome assignment of a human gene encoding a novel phosphatidylinositol-3 kinase. DNA Res 4: 355-358

Ueki N, Oda T, Kondo M, Yano K, Noguchi T, Muramatsu M (1998) Selection system for genes encoding nuclear-targeted proteins. Nat Biotechnol 16: 1338-1342

Vaisse C, Halaas JL, Horvath CM, Darnell JE Jr, Stoffel M, Friedman JM (1996) Leptin activation of Stat3 in the hypothalamus of wildtype and ob/ob mice but not db/db mice. Nat Genet 14: 95-97

Zhong Z, Wen Z, Darnell JE Jr (1994) Stat3: a STAT family member activated by tyrosine phosphorylation in response to epidermal growth factor and interleukin-6. Science 264: 95-98 